\title{
Hydrogel Nanospike Patch as a Flexible Anti- Pathogenic Scaffold for Regulating Stem Cell Behavior
}

Sunho Park,,$+\perp$ Hyun-Ha Park,,$+\perp$ Kahyun Sun,,+ Yonghyun Gwon,$^{\dagger}$ Minho Seong,,+ Sujin Kim, ${ }^{\dagger}$ Tae-Eun Park, ${ }^{\S}$ Hoon Hyun, $₫$ Yun-Hoon Choung, ${ }^{\#}$ Jangho Kim, ${ }^{* \dagger}$ and Hoon Eui Jeong*,t

Department of Rural and Biosystems Engineering, Chonnam National University, Gwangju 61186, Republic of Korea

tDepartment of Mechanical Engineering, Ulsan National Institute of Science and Technology (UNIST), Ulsan 44919, Republic of Korea

§School of Life Science, Ulsan National Institute of Science and Technology (UNIST), Ulsan 44919, Republic of Korea

'Department of Biomedical Sciences, Chonnam National University Medical School, Gwangju 61469, Republic of Korea

\#Department of Otolaryngology, Ajou University School of Medicine, Suwon 16499, Republic of Korea

*Corresponding author

Email: hoonejeong@unist.ac.kr (H. E. Jeong)/ rain2000@jnu.ac.kr (J. Kim). 


\section{Evaluation of mechanical properties of the hydrogel nanospike array (hSPIKE) with} different geometries.

The spring constant of the hSPIKE can be obtained by the following equation ${ }^{1}$

$$
k=\frac{3}{64} \pi E \frac{D^{4}}{L^{3}} \quad(S 1)
$$

where $D, L$, and $E$ represent the diameter, length, and Young's modulus of the hSPIKE, respectively.

The effective shear modulus of the hSPIKE is given by ${ }^{1}$

$$
G_{\text {eff }}=\frac{3}{16}\left(\frac{D}{L}\right)^{2} f E
$$

where $f$ is the surface coverage of the nanospike.

\section{NIR fluorescent imaging}

To evaluate the delivery efficiency of the hSPIKE, the NIR fluorophores as the model drug (ESNF 10) were prepared. ${ }^{2}$ The NIR fluorophores were diluted with methanol and PBS (NIR fluorophores:methanol:PBS=1:4:5), and the diluted NIR fluorophores of $500 \mu$ were loaded on the flat substrates and the hSPIKE $(1 \mathrm{~cm} \times 1 \mathrm{~cm})$. The samples were dried in the vacuum oven overnight at room temperature. For in vitro test, the prepared samples were washed with $70 \%$ ethanol for three times, then it was washed with $1 \%$ PBS. The washed samples were immersed in the normal medium for 3 hours, and the cells $\left(1 \times 10^{5}\right.$ cells/samples $)$ were cultured for 3 hours and 3 days. The NIR signal was confirmed by laser confocal scanning microscope system (TCS SP5/AOBS/Tandem, Wetzla, Germany) at $750 \mathrm{~nm}$. For in vivo test, the prepared samples were injected into the skin of adult (6-week-old) male NCr nude (NCRNU) weighing approximately $25 \mathrm{~g}$ mice for $1 \mathrm{~min}$. The NIR signal was confirmed by Fluorescence-labeled Organism Bioimaging Instrument (FOBI, NeoScience, South Korea). 
Table S1. Types of different nanomaterials to interface with living cells and their characteristics.

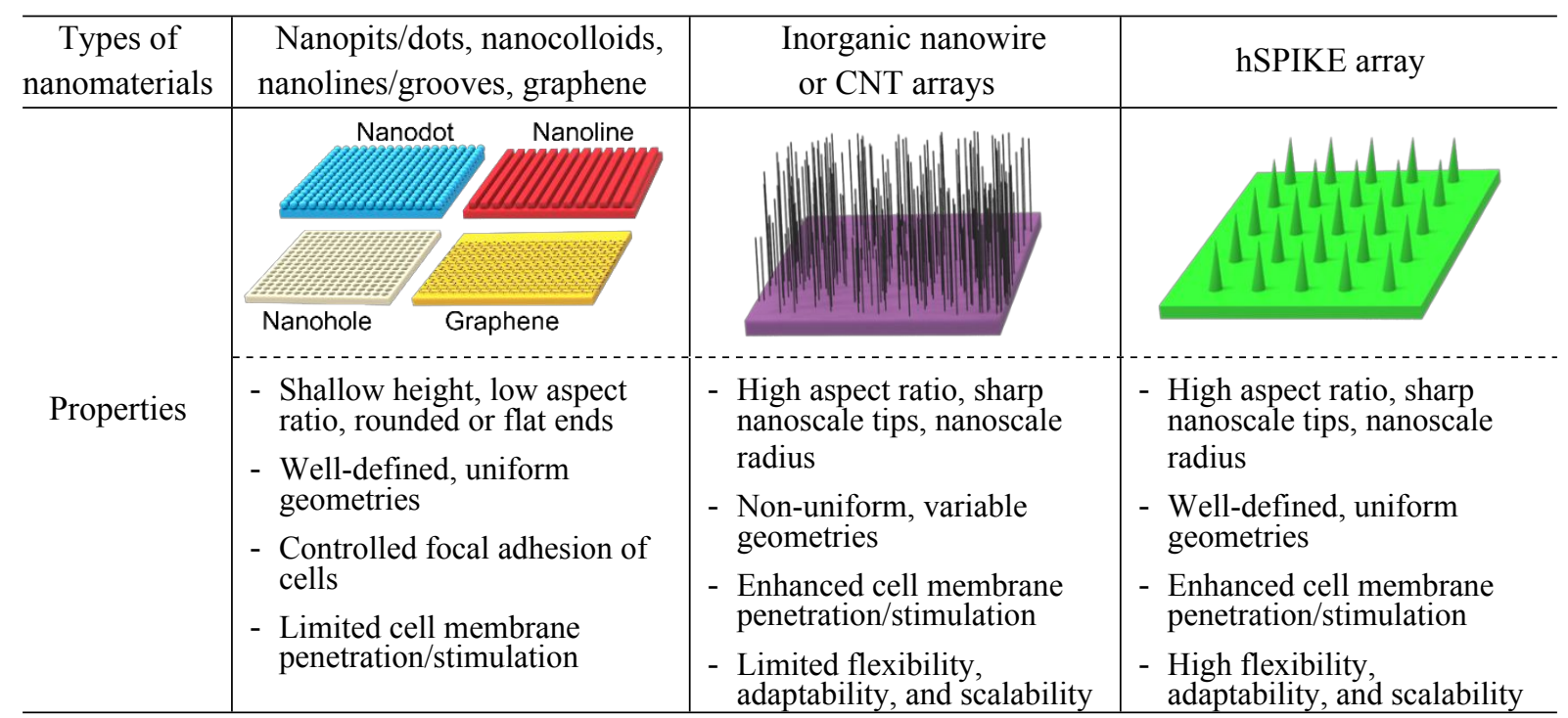


a

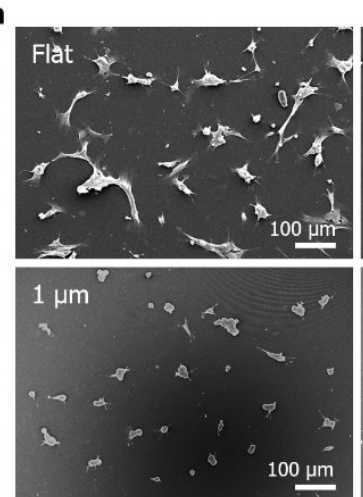

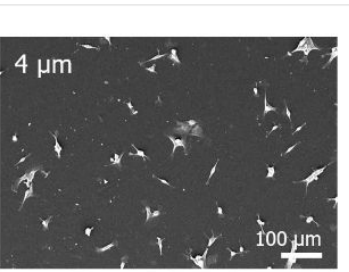

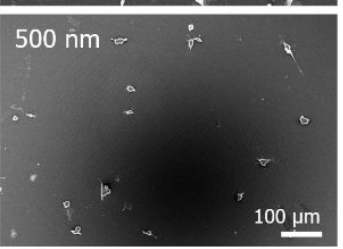

b
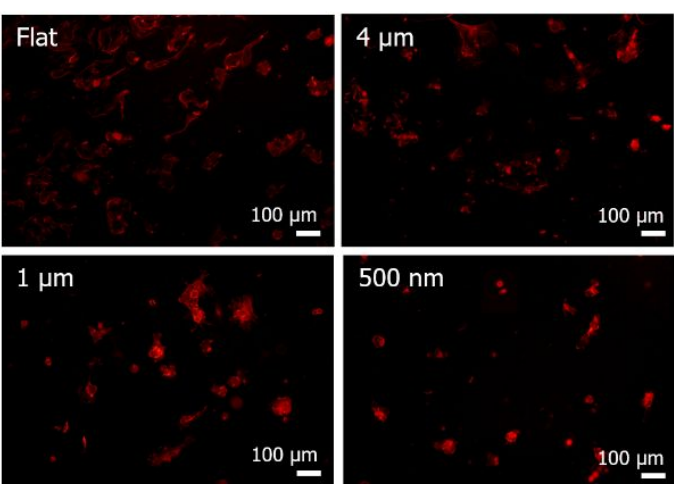

Figure S1. Cellular responses of DPSCs on hSPIKEs. (a) Representative SEM images of the morphologies of DPSCs on the hSPIKEs and the flat substrates. (b) Representative immunostaining images of F-action (red) of DPSCs on the hSPIKEs and flat substrates. 

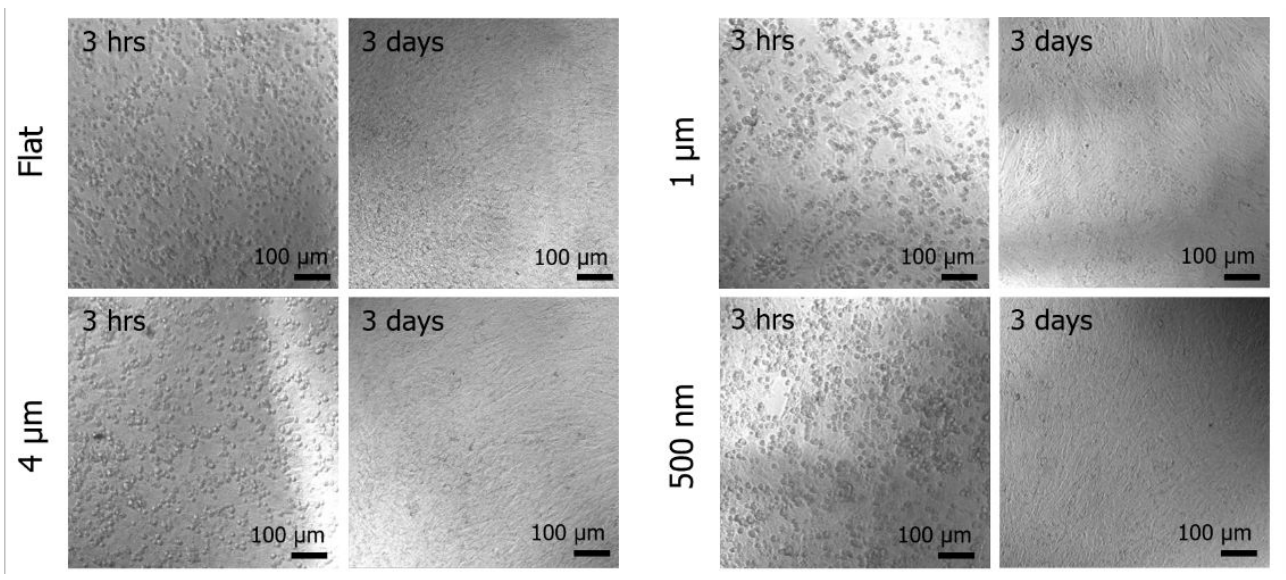

Figure S2. Representative microscopy images of cultured DPSCs on hSPIKEs and flat substrates for 3 hours and 3 days, showing that hSPIKEs can provide good environments for cell proliferation.

\section{References}


1. Rasmussen, C. H.; Reynolds, P. M.; Petersen, D. R.; Hansson, M.; McMeeking, R.M.; Dufva, M.; Gadegaard, N. J., Enhanced Differentiation of Human Embryonic Stem Cells toward Definitive Endoderm on Ultrahigh Aspect Ratio Nanopillars. Adv. Funct. Mater. 2016, 26, 815-823.

2. Kim, S. H.; Park, G.; Hyun, H.; Lee, J. H.; Ashitate, Y.; Choi, J.; Hong, G. H.; Owens, E. A.; Henary, M.; Choi, H. S., Near-Infrared Lipophilic Fluorophores for Tracing Tissue Growth. Biomed. Mater. 2013, 8, 014110. 